\title{
Neuropathic spinal arthropathy leading to spine disruption, spinal cord transection, and aortic displacement: brief case report
}

\author{
Lance L. Goetz $\mathbb{B}^{1,2} \cdot$ Sean McAvoy ${ }^{1,2} \cdot$ Kate Zakrzewski ${ }^{2}$ \\ Received: 30 July 2018 / Revised: 29 August 2018 / Accepted: 4 September 2018 \\ (c) International Spinal Cord Society 2018
}

\begin{abstract}
Introduction Charcot spinal arthropathy (CSA) is an infrequent but potentially devastating complication after spinal cord injury.

Case presentation We report a case of a man with longstanding T3 complete (AIS A) paraplegia who developed severe CSA with spine disruption and aortic displacement.

Discussion Acute management of this patient is described along with both conservative and surgical management considerations and challenges as described in other reports.
\end{abstract}

\section{Introduction}

Charcot joint, or neuropathic arthropathy, is a destructive degenerative joint process named after the famous French neurologist Jean-Martin Charcot. Charcot's initial subjects were persons suffering from lack of protective joint sensation due to syphilitic tabes dorsalis. Others before Charcot had described the history of the problem of neuropathic arthropathy, however [1, 2].

In the US, Charcot joints are frequently seen in persons with diabetes and severe peripheral polyneuropathy impairing joint protective sense at the ankle. Charcot joints, including Charcot spine, can occur in persons with spinal cord injury, and particularly in persons with syringomyelia, in any joint below the neurologic level of injury. Charcot spinal arthropathy (CSA) occurring after SCI has been reported by several authors. Standaert, Cardenas, and Anderson reported a series of five persons with complete thoracic paraplegia and Charcot spine occurring 6-31 years after SCI [3]. Grassner et al. [2] reported a series of four

Lance L. Goetz

Lancelgoetz@gmail.com

1 Spinal Cord Injury and Disorders Service, Hunter Holmes McGuire Veterans Affairs Medical Center, Richmond, VA, USA

2 Department of Physical Medicine and Rehabilitation, Virginia Commonwealth University, Richmond, VA, USA females, again with complete thoracic paraplegia with CSA occurring 17-39 years after SCI.

Here we present a case of a man who presented with painless crepitance and was found to have an enlarging Charcot mass with spinal disruption and displacement of the aorta anteriorly. A PubMed search using the terms "Charcot spine" AND "spinal cord injury" AND "aorta" revealed no results. Of articles found pertaining to Charcot spine after spinal cord injury, none reported aortic involvement. Therefore, although this may have occurred in others, this is, to our knowledge, the first report of aortic displacement by a Charcot spine bone mass.

\section{Case presentation}

The patient is a 69-year old man with a 48-year history of T3 AIS A paraplegia due to gunshot wound incurred during military service. He was admitted to our facility for routine annual SCI evaluation. He reported a 5-6-month history of painless low back crepitance, which he described as "clunking" occurring with truncal rotation and difficulty with transfers from his wheelchair. He denied any fevers, chills, night sweats, or weight loss.

He had not had any surgical fusion surgeries to his spine. He did have a bullet removed via T5-6 laminectomy due to "scar and rubbing" two years following his injury.

Plain radiographs of the thoracic spine were performed which revealed worsening erosion of the T10 and T11 vertebral endplates with pseudarthrosis and marked 


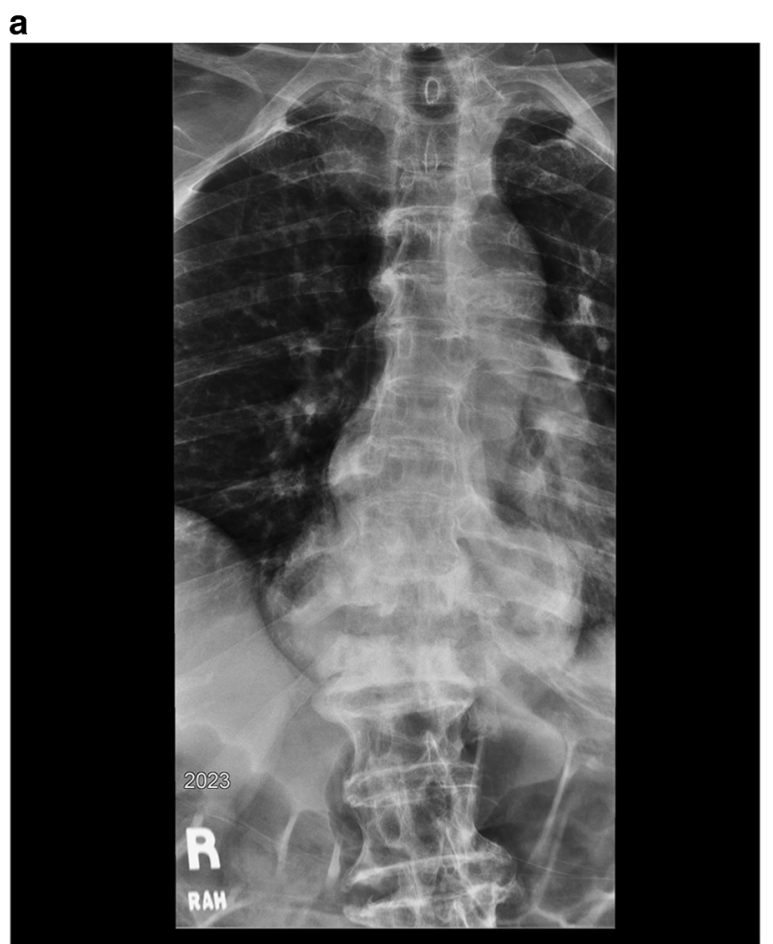

Fig. 1 a Radiograph of patient at annual evaluation showing worsening erosion of the T10 and T11 vertebral endplates with pseudarthrosis and marked paravertebral soft tissue swelling. b Radiograph of

paravertebral soft tissue swelling (Fig. 1a). Bony changes seen on plain chest radiographs taken two years prior (Fig. 1b) were much less severe, consistent with rapid progression after many years of relative stability.

Magnetic resonance imaging of the thoracic spine revealed a destructive process with pseudarthrosis, Grade 3 retrolisthesis at T10-T11, near obliteration of the spinal canal, marked bone formation, and a fluid collection consistent with neuropathic arthropathy or chronic discitis-osteomyelitis (Fig. 2a-d). The mass measured nearly $9 \mathrm{~cm}$ anteroposterior by $13 \mathrm{~cm}$ width by $8 \mathrm{~cm}$ craniocaudally. Notably, the extensive pre-vertebral bone formation caused displacement and deformation of the abdominal aorta anteriorly.

Following MRI, neurosurgery and infectious disease (ID) specialists were consulted for assistance with management. ID noted relevant lab values, including Westergren ESR of $10 \mathrm{~mm} / \mathrm{h}$ and C-reactive protein of $1.61 \mathrm{mg} / \mathrm{dL}$. Given radiology MRI report that findings "may represent infection" they recommended CT guided bone biopsy to rule out an infectious etiology. This was performed by the interventional radiology (IR) service. The sample yield was small, but gram stain and culture were negative, consistent with a noninfectious process and suspected Charcot spine. The neurosurgery consultants considered the patient for operative intervention with a long segment fusion with both anterior and posterior instrumentation. They discussed the

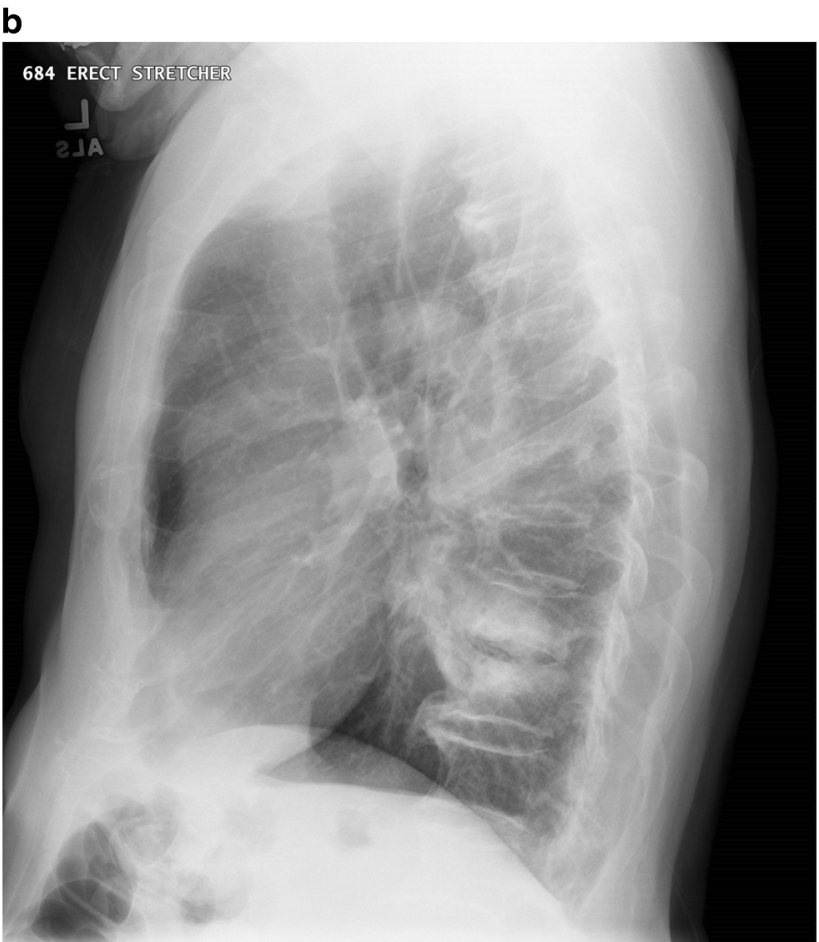

patient two years before annual evaluation, showing progressive sclerosis and lateral syndesmophyte formation at T10-T11

risks of such a surgery in detail, including blood loss, infection, pulmonary compromise, hardware failure, and a long recovery time, with the patient and our team. Conversely, risks of non-operative management include progression of destabilized spine leading to additional injury such as a conus lesion. As part of operative considerations, cardiothoracic surgery was consulted to assess risk of operative versus non-operative management in terms of aortic erosion or compromise. Their opinion was that aortic complications were not a near-term risk.

The patient wanted time to consider spinal fusion surgery and recovery time and discuss it with family. He was discharged to home with plan for a follow-up appointment in Neurosurgery clinic to discuss surgery if desired. Pulmonary function tests (PFTs) were ordered to monitor for additional restriction of lung volumes, which could potentially necessitate surgical stabilization to restore upright spine posture.

Ultimately, the patient, along with consultants, opted for conservative care. We respected his right to choose, especially given his age. Bracing was discussed as an option. A thoracic lumbar sacral orthosis (TLSO) was felt to be too function limiting, but a corset with stays might provide an intermediate option to provide some support, but allow for mobility and reaching. The veteran politely declined any brace. Due to difficulty with transfers and wheelchair 
a

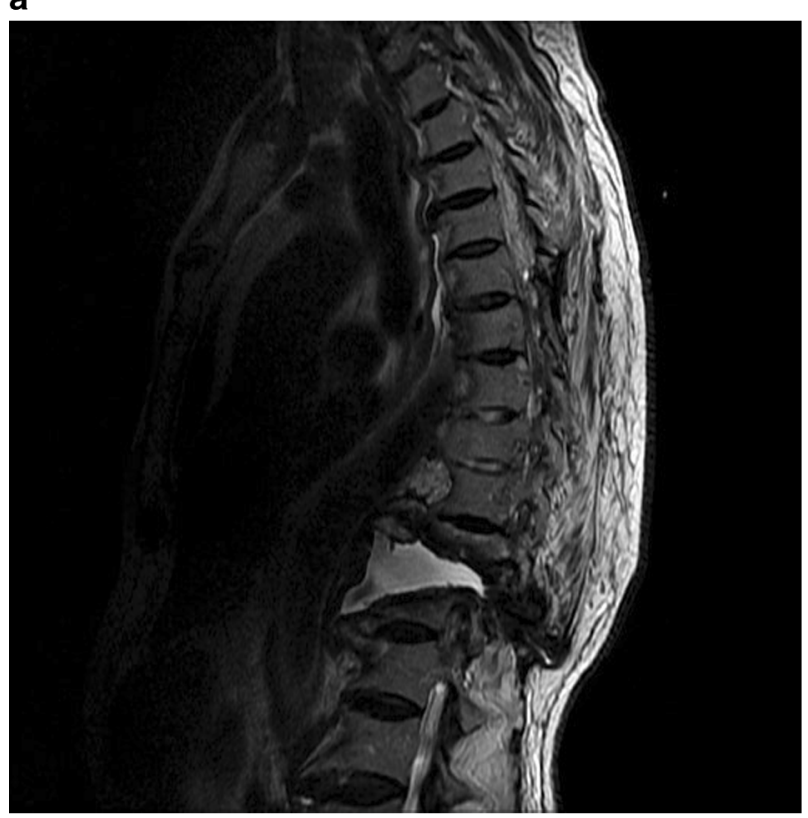

c

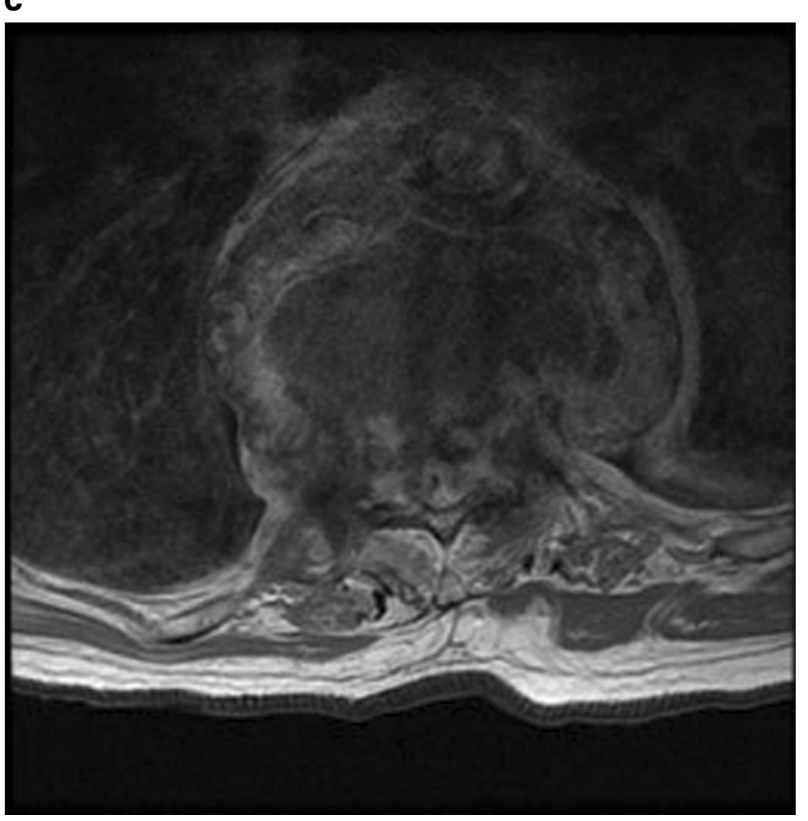

Fig. 2 a-d Magnetic resonance imaging of the thoracic spine with long standing chronic process at the T10-T11 vertebral level with exuberant bone formation, pseudarthrosis, and new grade 3 retrolisthesis

propulsion, he accepted a change to power mobility and use of a lift for transfers.

\section{Discussion}

This is the first report, to our knowledge, of aortic displacement secondary to severe neuropathic arthropathy of the spine. We suspect that this phenomenon is not rare, but it has not been discussed in the literature, and the likelihood b

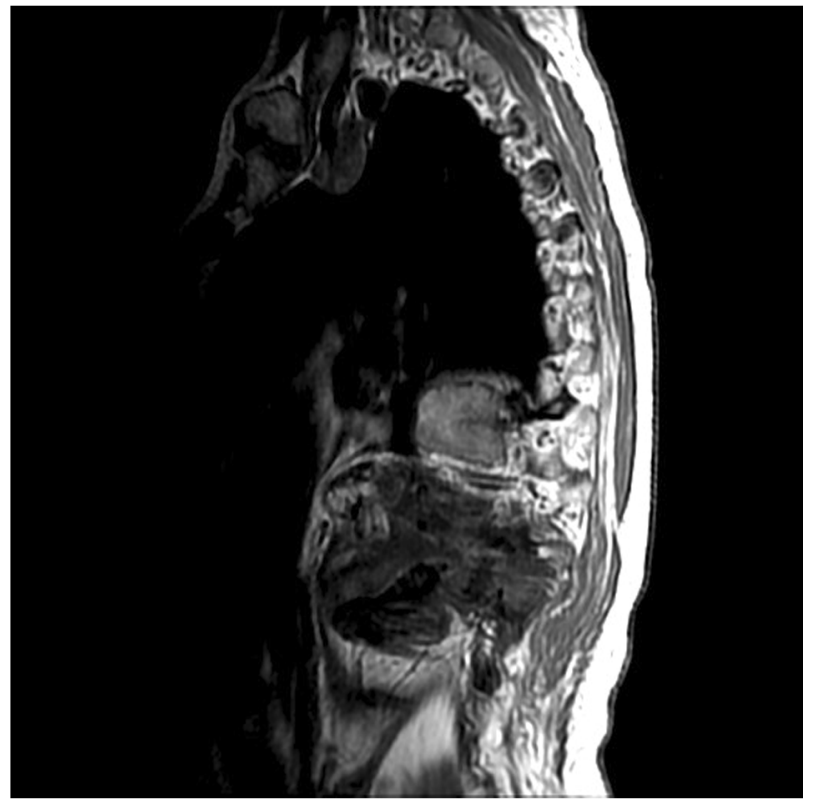

d

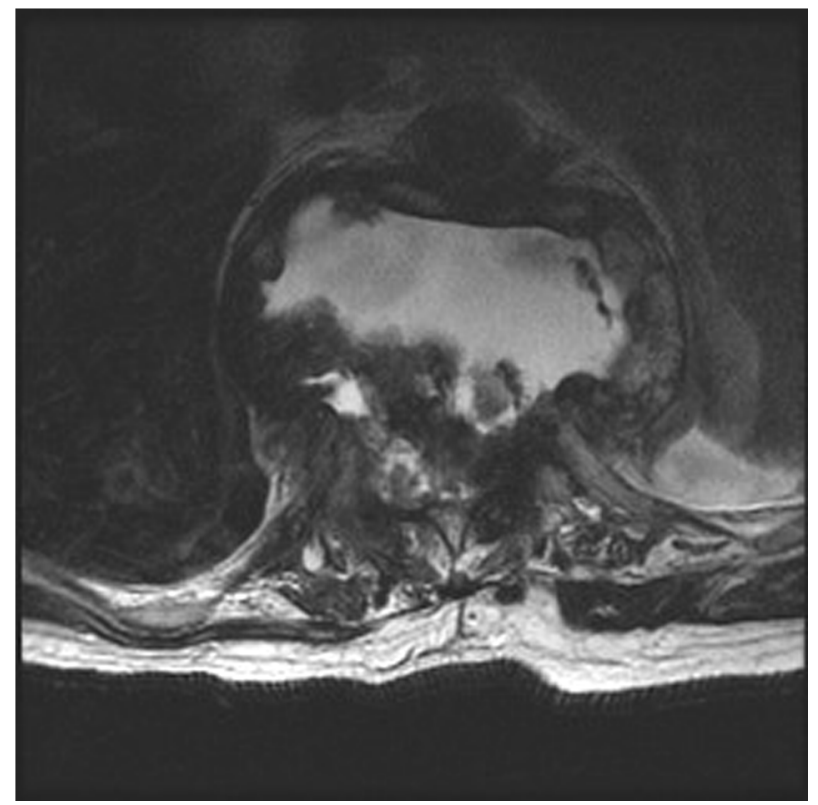

resulting in cord transection. The space between the destroyed T10 and T11 vertebral bodies is fluid-filled with new peripheral bone formation measuring $8.8 \mathrm{~cm} \mathrm{AP} \times 12.2$ transverse and $7.8 \mathrm{~cm}$ craniocaudal

of aortic complications is not clear. The location and health of the aorta and spinal level of the Charcot mass may be important factors, but this is not known. For example, would an atherosclerotic and relatively inflexible aorta be at risk for rupture? Long-term follow-up of persons treated with conservative care would be necessary to assess long term outcome.

In addition to aortic displacement, as seen in this case, untreated CSA can cause several complications. It has been noted to cause autonomic dysreflexia (AD) in susceptible 
persons $[4,5]$ and should be considered in the differential diagnosis, especially when other more obvious causes have been ruled out.

Clinical diagnosis of CSA is made somewhat more difficult due to the variable length to presentation after initial neurologic damage, and the limited symptoms given preexisting neurologic deficits. Time between date of SCI and appearance of CSA is reported as early as 6 years but is generally longer. In a systemic review, the mean time between onset of neurological impairment and the diagnosis of Charcot spine was $17.3 \pm 10.8$ years. The majority of cases develop damage below T10, which correlates to spine mobility and affect one spinal joint [6].

CSA is also a diagnostic challenge because its imaging features are similar to those of spinal conditions such as discitis-osteomyelitis, osseous tuberculosis, hemodialysisrelated spondyloarthropathy, and pseudarthrosis [7]. Differential diagnoses include chronic infections or tumor, which also present with bone erosion, osteophytosis, and narrowing of the intervertebral space and paravertebral mass. Radiographically, CSA presents with profound disc degeneration, vertebral erosion with osteosclerosis, hyperostosis, and destructive facet joint changes [2].

Charcot spine develops in the setting of injured or inflamed tissue that lacks intact sensory feedback. This is often seen within operated areas, particularly following laminectomies, as loss of posterior stabilizing support increases stress on remaining spinal elements. As arthropathy progresses, damage to all three columns can induce instability and dislocation.

There is no straightforward or universally acceptable management. Immobilization of the Charcot joint is essential to treat CSA and prevent neurologic sequela. Conservative management of Charcot arthropathy includes immobilization of the affected join and weight bearing restrictions. However, surgical intervention is almost always eventually required.

Surgery serves to stabilize the affected spinal segment by creating a fusion, often with necrotic or inflamed tissue debridement via anterior approach. Surgical options considered with CSA include anterior and posterior circumferential fusion with extensive anterior debridement, posterior instrumentation, and autogenous bone graft. Posterior fixation allows mechanical stability, while biological secondary stability is dependent on bone fusion [8]. In a four-case review, no serious post-operative complication or instrumentation failure was observed [9].

Surgery is considered first line treatment; however, it can have many potential complications including hardware loosening, wound healing delays and development of additional Charcot lesions [10]. While short-term studies show success in sagittal balance, postoperative fusion, and pain reduction [11], long-term follow up reveals several postoperative complications. In a long-term follow-up of eight patients 14 years following surgery, post-operative complications included loss of functional mobility, osteomyelitis, pressure ulcers, and formation of a Charcot lesion leading to revision operation. Fusion in Charcot spine is prolonged, with patients often requiring multiple attempts. This is likely due to complicated healing of a sclerotic, avascular lesion [12].

Systemic extension of stabilization into the pelvis reduces risk of new arthropathy, however surgery is very complex and may lead to function limits in mobility and transfers. Hip tightness can cause increased compensatory lumbar motion when sitting or supine and will impair postoperative fusion. Correctable causes of hip joint contracture, such as hypertonicity or heterotopic ossification, should be addressed prior to spinal fusion [3].

The issues with this patient highlight complications that can occur with CSA, and challenges in management. Persons diagnosed with this problem require close long-term follow-up.

\section{Compliance with ethical standards}

Conflict of interest The authors declare that they have no conflict of interest.

\section{References}

1. Gupta R. A short history of neuropathic arthropathy. Clin Orthop Relat Res. 1993;296:43-9.

2. Grassner L, Geuther M, Mach O, Bühren V, Vastmans J, Maier D. Charcot spinal arthropathy: an increasing long-term sequel after spinal cord injury with no straightforward management. Spinal Cord Ser Cases. 2015;1:15022.

3. Standaert C, Cardenas DD, Anderson P. Charcot spine as a late complication of traumatic spinal cord injury. Arch Phys Med Rehabil. 1997;78:221-5.

4. Zyck S, Toshkezi G, Pizzuti J, Marawar S. Four-rod instrumentation for treatment of Charcot spinal arthropathy causing autonomic dysreflexia: case report and literature review. Cureus. 2016;8:e850.

5. Morita M, Iwasaki M, Okuda S, Oda T, Miyauchi A. Autonomic dysreflexia associated with Charcot spine following spinal cord injury: a case report and literature review. Eur. Spine J. 2010;19 (Suppl 2):S179-82.

6. Barrey C, Massourides H, Cotton F, Perrin G, Rode G. Charcot spine: two new case reports and a systematic review of 109 clinical cases from the literature. Ann Phys Rehabil Med. 2010;53:200-20.

7. Ledbetter LN, Salzman KL, Sanders RK, Shah LM. Spinal Neuroarthropathy: Pathophysiology, clinical and imaging features, and differential diagnosis. Radiographics. 2016;36:783-99.

8. Proietti L, Pola E, Nasto LA, Scaramuzzo L, Logroscino CA. Onset of a Charcot spinal arthropathy at a level lacking surgical arthrodesis in a paraplegic patient with traumatic cord injury.Eur Spine J. 2010;19(Suppl 2):S83-6.

9. Suda Y, Shioda M, Kohno H, Machida M, Yamagishi M. Surgical treatment of Charcot spine. J Spinal Disord Tech. 2007;20:85-8. 
10. Aebli N, Pötzel T, Krebs J. Characteristics and surgical management of neuropathic (Charcot) spinal arthropathy after spinal cord injury. Spine J. 2014;14:884-91.

11. Vialle R, Mary P, Tassin J-L, Parker F, Guillaumat M. Charcot's disease of the spine: diagnosis and treatment. Spine. 2005;30: E315-22.
12. Haus BM, Hsu AR, Yim ES, Meter JJ, Rinsky LA. Long-term follow-up of the surgical management of neuropathic arthropathy of the spine. Spine J. 2010;10:e6-16. 